Décadrages Décadrages

cinéma, à travers champs Cinéma, à travers champs

$14 \mid 2009$

Cinéma et migration

\title{
Cinéma suisse et migration : une tentative de filmographie
}

\section{(2) OpenEdition \\ Journals}

Édition électronique

URL : https://journals.openedition.org/decadrages/282

DOI : $10.4000 /$ decadrages. 282

ISSN : 2297-5977

Éditeur

Association Décadrages

Édition imprimée

Date de publication : 1 avril 2009

Pagination : 9-12

ISBN : 978-2-9700582-9-8

ISSN : 2235-7823

Référence électronique

"Cinéma suisse et migration : une tentative de filmographie », Décadrages [En ligne], 14 | 2009, mis en ligne le 10 avril 2010, consulté le 03 avril 2022. URL : http://journals.openedition.org/decadrages/282 ; DOI : https://doi.org/10.4000/decadrages.282 


\section{Cinéma suisse et migration : une tentative}

\section{de filmographie}

- Füsilier Wipf, Leopold Lindtberg, 1938

- L'oasis dans la tourmente, Arthur Porchet, 1941

- Landammann Stauffacher, Leopold Lindtberg, 1941

- Marie-Louise, la petite Française, Leopold Lindtberg, 1941

- Die Letzte Chance, Leopold Lindtberg, 1945

- La Suisse s'interroge, Henry Brandt, 1964

- Siamo Italiani, Alexander Seiler, June Kovach, Robert Gnant, 1964

- Braccia si, uomini no, Peter Amman, René Burri, 1970

- 24 su 24, Villi Hermann, 1970

- Lo Stagionale, Alvaro Bizzari, 1971

- Storia di confine, Bruno Soldini, 1972

- Le Train rouge, Peter Amman, 1973

- Le Retour d'Afrique, Alain Tanner, 1973

- Il Rovescio della medaglia, Alvaro Bizzari, 1974

- Die Auslieferung, Peter von Gunten, 1974

- Cerchiamo per subito operai, offriamo..., Villi Hermann, 1974

- Arbeiterehe, Robert Boner, 1974

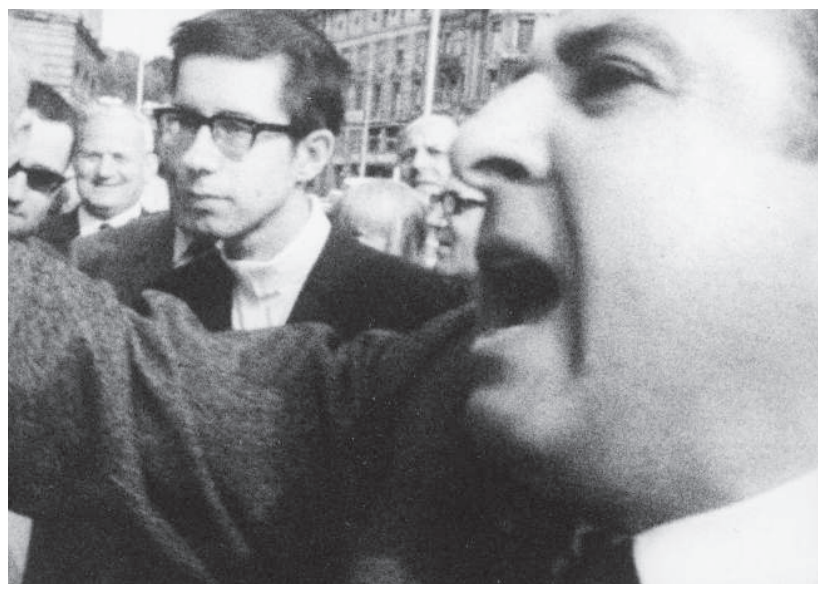

Braccia si, uomini no, Peter Amman, René Burri, 1970

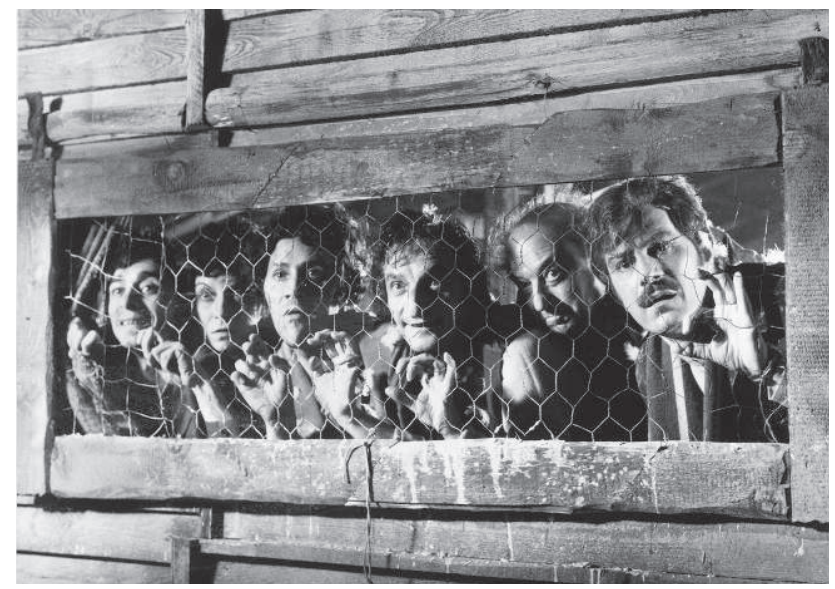

Pane e cioccolata, Franco Brusati, 1974 


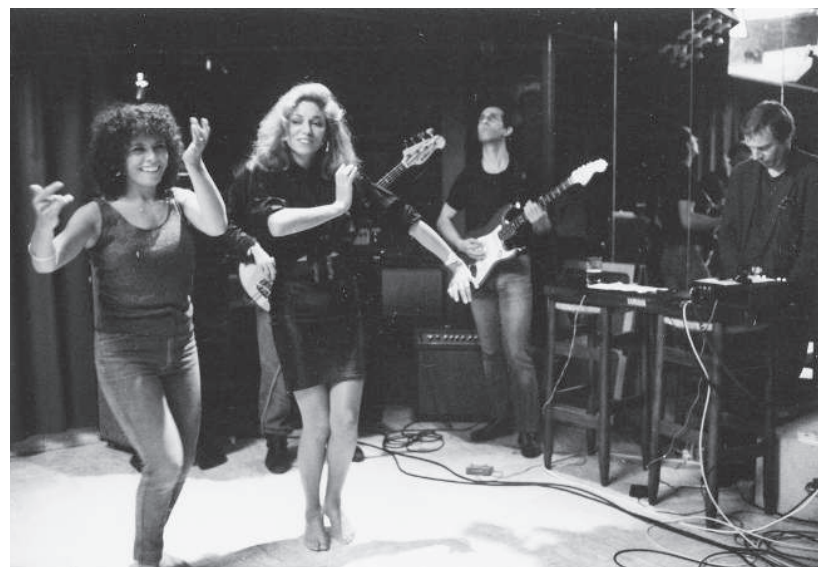

No man's land, Alain Tanner, 1984

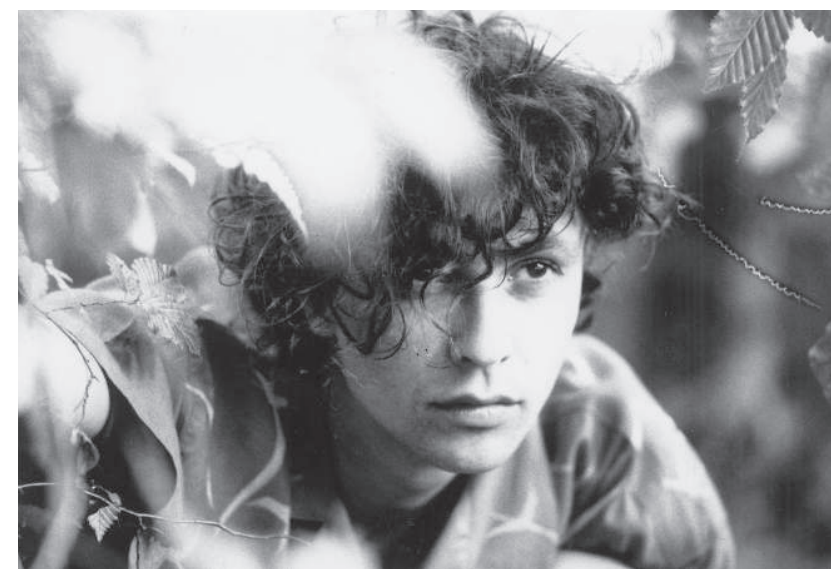

Filou, Samir, 1988

- Pane e cioccolata, Franco Brusati, 1974

- Le Milieu du monde, Alain Tanner, 1974

- Buseto, Remo Legnazzi, 1974

- Jonas qui aura 25 ans en l'an 2000, Alain Tanner, 1975

- Pagine di vita dell'emigrazione, Alvaro Bizzari, 1977

- Bern Transit, Ulrich Schweizer, 1977

- San Gottardo, Villi Hermann, 1977

- Die Schweizermacher, Rolf Lyssy, 1978

- Emigrazione, Nino Jacusso, 1978

- Ritorno a casa, Nino Jacusso, 1980

- Il Valore della donna è il suo silenzio, Gertrud Pinkus, 1980

- Das Boot is voll, Markus Imhoof, 1981

- Zweiter Anfang, Remo Legnazzi, 1981

- Unsere Eltern haben den Ausweis "C», Eduard Winiger, 1982

- Une danse pour l'exil, Jean-Daniel Bloesch, 1984

- No man's land, Alain Tanner, 1984

- Das kalte Paradies, Bernard Safarik, 1986

- My Mother Is in Sri Lanka, Remo Legnazzi, Jürg Neuenschwander, 1987

- Filou, Samir, 1988

- Fluchtland, Markus Barmettler, Giorgio Andreoli, Madeleine Hadorn, 1989

- Reise der Hoffnung, Xavier Koller, 1990

- Touchol, Alvaro Bizzarri, 1990

- Kebab \& Rosoli, Karl Saurer, Elena M. Fischli, 1992

- Das grosse Mitte-Land, Samir, 1993 

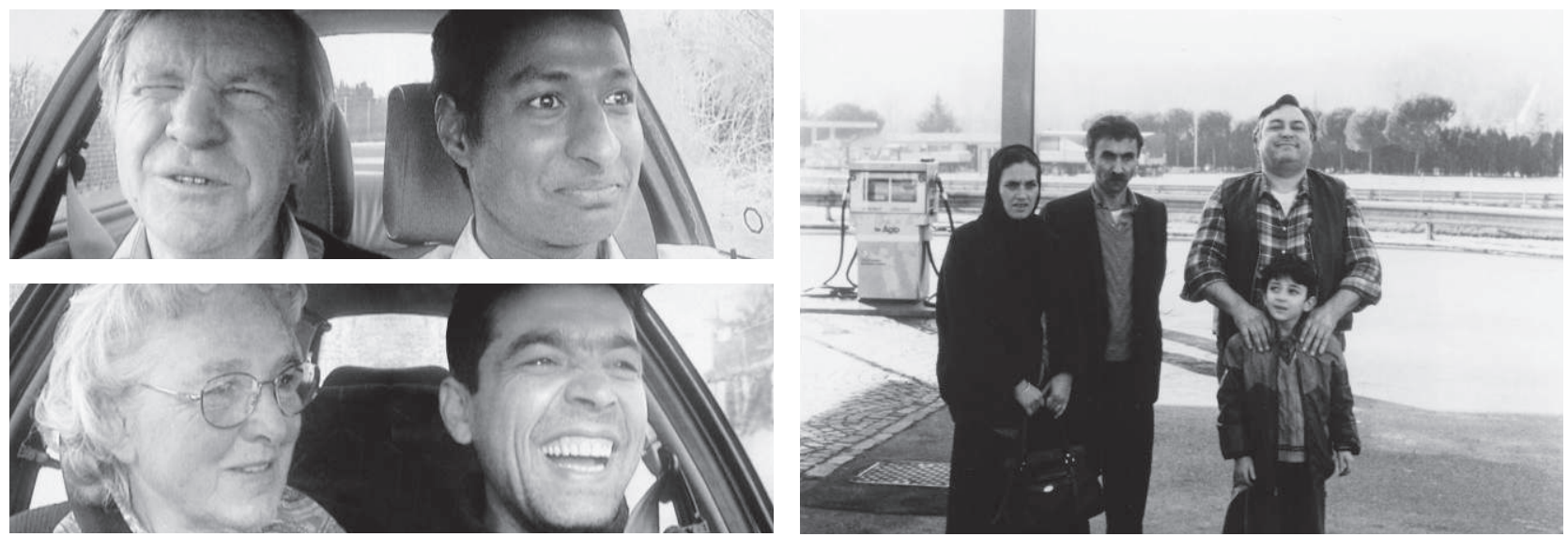

La bonne conduite, Jean-Stéphane Bron, 1999

Reise der Hoffnung, Xavier Koller, 1990

- Babylon 2, Samir, 1993

- Album de famille, Fernand Melgar, 1994

- Letters to Srebenica, Daniel von Aarburg, 1995

- They Teach Us How to Be Happy, Peter von Gunten, 1996

- Clandestins, Nicolas Wadimoff, Denis Chouinard, 1997

- Heimkebr in zerrissene Land, Marianne Pletscher, 1997

- La Nuit du veilleur, Dinu Musale, 1997

- Classe d'accueil, Fernand Melgar, 1998

- La bonne conduite, Jean-Stéphane Bron, 1999
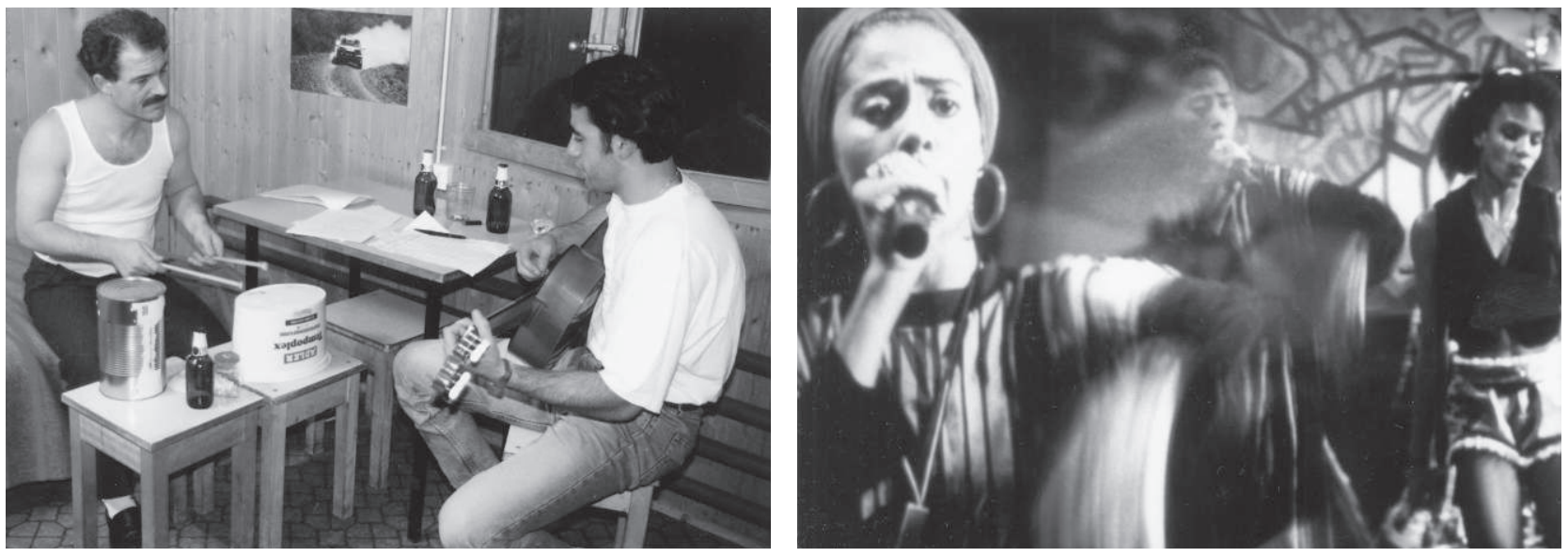

Touchol, Alvaro Bizzarri, 1990

Babylon 2, Samir, 1993 


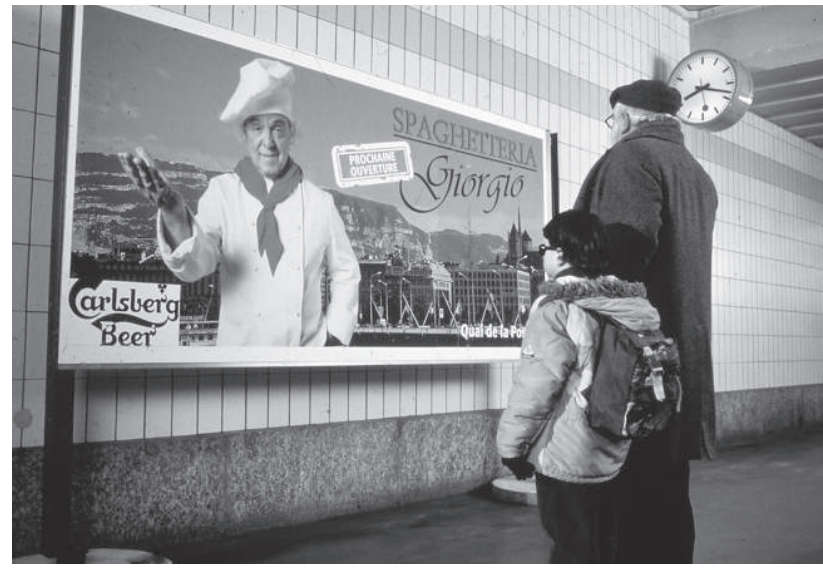

Azzurro, Denis Rabaglia, 2001

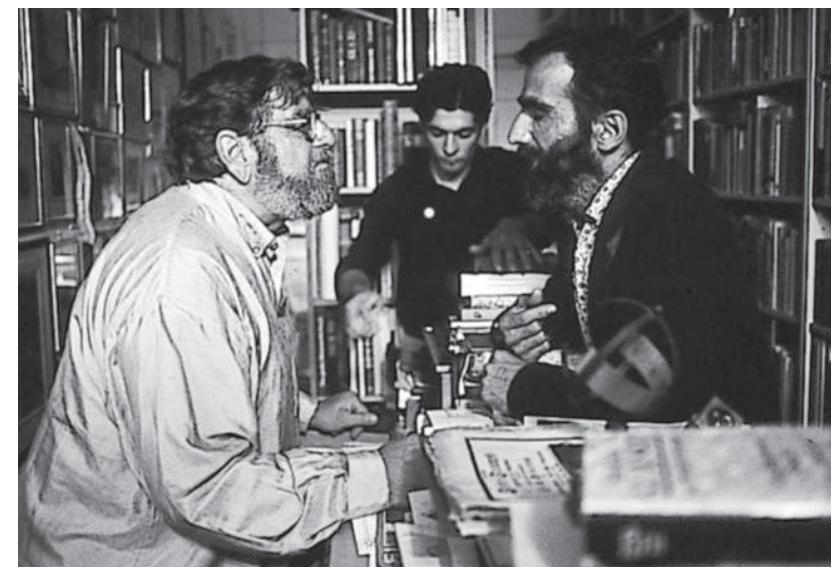

Escape to Paradise, Nino Jacusso, 2001

- Closed Country, Kaspar Kasics, 1999

- ID Swiss, collectif, 1999

- Transito, Nino Jacusso, 2000

- Azzurro, Denis Rabaglia, 2001

- Escape to Paradise, Nino Jacusso, 2001

- Clandestins - Le Voyage infernal, Jean-Paul Mudry, Roland Tillmanns, 2001

- Pas les flics, pas les noirs, pas les blancs, Ursula Meier, 2002

- Profil bas, Nathalie Flückiger, 2005

- Zwischen den Welten, Yusuf Yesilöz, 2006

- Das Fräulein, Andrea Staka, 2006

- Swiss Sans-Papiers, Andreas Hoessli, 2006

- 1956-2006: cinquante ans de regards sur l'asile en Suisse, Jean-Denis Borel, Raffaele Poli, 2007

- La Forteresse, Fernand Melgar, 2008

\section{Courts métrages}

- Les petites Illusions, Markus Imhoof, 1991 (in Le Film du cinéma suisse)

- Mersal El Hawa, Elie Khalifé, 1992

- Bel Canto, François Bovy, 1994

- Senza, Horst Züger, 1994

- Nascondiglio - Das Versteck, Roberto Di Valentino-Vasques, 1995

- Asyl, Ina Volmer, 1995

- Olmeye Yatmak - Und sie legte sich zum sterben nieder, Elsen Isik, 1997

- Samb et le commissaire, Olivier Sillig, 1997 